University of Texas at El Paso

ScholarWorks@UTEP

6-2009

\title{
Measurement's result and its error as fuzzy variables: background and perspectives
}

Gennady N. Solopchenko

Konstantin K. Semenov

Vladik Kreinovich

The University of Texas at El Paso, vladik@utep.edu

Leon Reznik

Follow this and additional works at: https://scholarworks.utep.edu/cs_techrep

Part of the Computer Engineering Commons

Comments:

Technical Report: UTEP-CS-09-18

Short version published in the Proceedings of the 9th International Symposium on

Measurement Technology and Intelligent Instruments ISMTII'2009, St. Petersburg, Russia, June 28 - July 2, 2009, pp. 4-132 - 4-136; full version published in Key Engineering Materials, 2010, Vol. 437, pp. 487-491.

\section{Recommended Citation}

Solopchenko, Gennady N.; Semenov, Konstantin K.; Kreinovich, Vladik; and Reznik, Leon, "Measurement's result and its error as fuzzy variables: background and perspectives" (2009). Departmental Technical Reports (CS). 45.

https://scholarworks.utep.edu/cs_techrep/45

This Article is brought to you for free and open access by the Computer Science at ScholarWorks@UTEP. It has been accepted for inclusion in Departmental Technical Reports (CS) by an authorized administrator of ScholarWorks@UTEP.For more information, please contact Iweber@utep.edu. 


\title{
Measurement's result and its error as fuzzy variables: background and perspectives
}

Guennady N. Solopchenko, Konstantin K. Semenov

Saint-Petersburg State Polytechnical University, 29, Polytechnicheskaya str., Saint-Petersburg, 195251, Russia Email: g.n.solopchenko@mail.ru semenov.k.k@gmail.com
Vladik Kreinovich

Department of Computer Science

University of Texas at El Paso,

El Paso, TX 79968-0518

Email: vladik@cs.utep.edu

Leon Reznik

Department of Computer Science

Rochester Institute of

Technology

102 Lomb Memorial Drive,

Rochester, NY 14623-5608

Email: lr@cs.rit.edu

\begin{abstract}
The possibility of using fuzzy variables for describing measurands and their error characteristics is investigated. The elementary arithmetic operations within the limits of such representation are considered.
\end{abstract}

Keywords: fuzzy variables, measurands, measurement results and characteristics of their errors, result's processing.

\section{Introduction}

Planning and performing measurements in modern measuring intelligenent systems (MIS) are carried out in the following sequence:

a) formation of the measurand's apriori model;

b) univocal analog-to-digital converting and entering the measurement's information into computer memory;

c) mathematical processing of measurements results corresponding to MIS software;

d) possible using of databases and expert systems (artificial intelligence techniques) for MIS adjstment or self-adjustment of if required;

e) presenting of measurements, their processing results and their errors characteristics.

In stages a) and d) the information is represented generally as fuzzy variables and its uncertainty - as a segment of the of the membership function cut at the specified level of degree of belief. Errors, which are generated by interactions between measuring instruments and objects of measurements, can be described in the same way.

Errors (uncertainty), which are caused by inaccuracy of physical transformations of a measured signal, are given by their statistical characteristics. As a rule noone normalizes or estimates errors, which are generated by computational software, except for rare occasions, when these calculations are statistical data processing.

Expressing the errors with the statistical models doesn't correspond to the data representation as fuzzy numbers. In the present report an attempt to represent measurements results and its errors (uncertainties) as fuzzy variables is made.

The main attention is given to the search for a practical implementation of mathematical operations with fuzzy variables with minimal complexity. An ultimate goal is the development of "self-verified" software for MIS that can provide not only an actual result but also an estimate of characteristics of its errors at the end of the execution cycle.

The Law of the Russian Federation "On supporting of measurements' unanimity» demands this. Also this principle corresponds to the requirements of the international recommendations towards expressing an uncertainty in measurements. 
Attempts to create such "self-verified" software have been already made. Their basic drawback is an application of the interval arithmetic, which gives too overestimated characteristics of error (which cannot satisfy the user in all cases).

\section{Background}

The main principles of the fuzzy numbers' theory are described, for example, in [2].

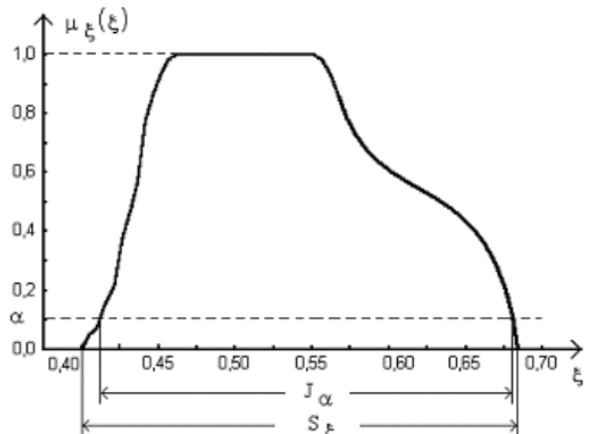

Figure . 1. Membership function of fuzzy variable $\xi$.

Marked: degree of membership $\alpha$, nested interval $J_{\alpha}$, support $S_{\xi}$ of fuzzy variable $\xi$.
Let $x$ and $y$ be fuzzy numbers, $S_{x}$ and $S_{y}$ be their supports, $z$ is a result of an arithmetical operation, $\otimes$ is symbol of arithmetical operation. Then membership function (MF) of number $z$ can be determined by rule:

$$
\mu_{z}(z)=\sup _{\substack{z=x \otimes y \\ x \in S_{x}, y \in S_{y}}}\left\lfloor\mu_{x}(x) \cdot \mu_{y}(y)\right] .
$$

As the membership function of measurand we offer to use symmetric curvilinear trapezium, which has height equal to one and presents information about characteristics of systematic error and total error:

$$
\mu_{z}(z)=\left\{\begin{array}{cc}
\exp \left(-(z-a)^{2} /\left(2 \cdot \sigma^{2}\right)\right), & a-3 \cdot \sigma<z \leq a \\
1, & a<z<b \\
\exp \left(-(z-b)^{2} /\left(2 \cdot \sigma^{2}\right)\right), & b+3 \cdot \sigma>z \geq b
\end{array}\right.
$$

This format of membership functions allows satisfying to the following conditions $(*)$ :

- averaging-out of multiple measurements results, which are characterized by only Type A uncertainty, must bring to reducing an standard uncertainty in a reverse proportion to the square root of averaged measurements results;

- input data and estimation of final result's error must be shown by the standard way: in the form of intervals of Types A and B uncertainties or in the form of combined uncertainty;

- intervals corresponded to Types A and B uncertainties of measurements results must be unequivocally separated / taken from such format of membership function.

On picture 2a membership function of fuzzy interval is given and intervals for Types A and B uncertainties components are marked. When it is no Type A uncertainty (random component of error), the membership function has a form of rectangle (fig. $2 \mathrm{~b}$ ). When it is no Type B uncertainty (error systematic component) - a form of Gaussian density (fig. 2c):

$$
\mu_{x}(x)=\exp \left(-\frac{x^{2}}{2 \cdot \sigma^{2}}\right) \text {. }
$$
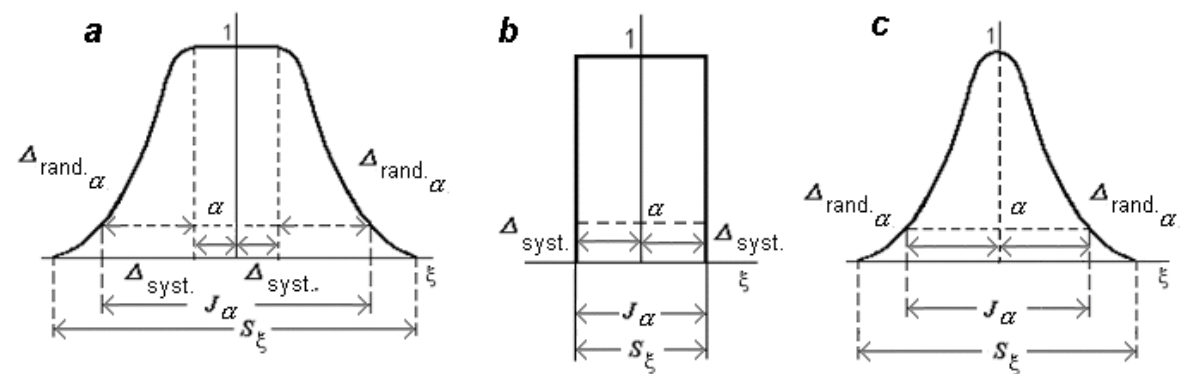

Figure. 2. Membership function of measurand, which is given with characteristics of its error:

$a$-presence of Types A and B uncertainties (systematic and random components of error); $b$ - absence of Type A uncertainty (random component of error); $c$ - absence of Type B uncertainty (systematic component of error).

We can compute the parameter $\sigma$ depending on specified level of degree $\alpha$ by formula

$$
\sigma=\Delta_{\text {rand } . \alpha} / \sqrt{-2 \cdot \ln (\alpha)} \text {. }
$$


The coordinates of points $S_{\xi}$ for case of fig 2a we can compute as $(-4 \cdot \sigma, 0)$ and $(4 \cdot \sigma, 0)$ or $(-3.5 \cdot \sigma, 0)$ and $(3.5 \cdot \sigma, 0)$.

Satisfaction of the second requirement of the list $(*)$ follows from possibility of presenting fuzzy number $\xi$, which reflects results of direct measurements and whose MF is organized as in case on fig. 2a, as summa $\xi=\xi_{\text {rand. }}+\xi_{\text {syst. }}$ Here the fuzzy number $\xi_{\text {rand. }}$ has MF of the form given on fig. 1c, and described only Type A uncertainty (random part of error) and the fuzzy number $\xi_{\text {syst. }}$ has MF of sort, which is shown on fig. 1b, and presents only Type B uncertainty (systematic part of error).

It is necessary to know or estimate $\Delta_{\text {rand. }}$ and $\Delta_{\text {syst. }}$ and input it to specialized computer software to allow them to present membership function of measurements results.

Presented formalization methodology and software allow to carry out processing of direct measurements' results, accompanied by standard characteristics of errors, in the standard way.

\section{Operations on measured variables presented as fuzzy numbers}

In this article it is accepted to adhere formula (1) when evaluating elementary operations with fuzzy variables.

Calculating fuzzy intervals according to formula (1) is a very time consuming operation. This produces natural aspirations for its modifications in order to reduce the required resource consumption. We will begin optimization with operation of adding $z=x+y$.

For this purpose we carry out the maximum of expression $\max _{x+y=z}\left[\mu_{x}(x) \cdot \mu_{y}(y)\right]=\max _{x+y=z}\left[e^{-\frac{\left(x-x_{0}\right)^{2}}{2 \cdot \sigma_{x}^{2}}} \cdot e^{-\frac{\left(y-y_{0}\right)^{2}}{2 \cdot \sigma_{y}^{2}}}\right]$. Required maximum is reached in the point of maximum of function $f(x, y)=-\frac{\left(x-x_{0}\right)^{2}}{2 \cdot \sigma_{x}^{2}}-\frac{\left(y-y_{0}\right)^{2}}{2 \cdot \sigma_{y}^{2}}$. We make substitution $y=z-x$ to determine the value of $x_{\max }$. Then

$$
\begin{aligned}
& \varphi(x, z)=f(x, z-x)=-\frac{\left(x-x_{0}\right)^{2}}{2 \cdot \sigma_{x}^{2}}-\frac{\left(z-x-y_{0}\right)^{2}}{2 \cdot \sigma_{y}^{2}}, d \varphi(x, z) / d x=0 . \\
& \sigma_{y}^{2} \cdot\left(x-x_{0}\right)+\sigma_{x}^{2} \cdot\left(x+y_{0}-z\right)=0, x \cdot\left(\sigma_{y}^{2}+\sigma_{x}^{2}\right)=\sigma_{y}^{2} \cdot x_{0}+\sigma_{x}^{2} \cdot\left(z-y_{0}\right) \\
& x_{\max }=\arg \max _{x+y=z}\left[\mu_{x}(x) \cdot \mu_{y}(y)\right]=\frac{\sigma_{y}^{2} \cdot x_{0}+\sigma_{x}^{2} \cdot\left[z-y_{0}\right]}{\sigma_{x}^{2}+\sigma_{y}^{2}}
\end{aligned}
$$

Easy to see that this point is a real maximum for function $f(x, y)$.

We got the result in the form of equations $x_{\max }=\frac{\sigma_{y}^{2} \cdot x_{0}+\sigma_{x}^{2} \cdot\left[z-y_{0}\right]}{\sigma_{x}^{2}+\sigma_{y}^{2}}$ and $x_{\max }=\frac{\sigma_{y}^{2} \cdot x_{0}+\sigma_{x}^{2} \cdot\left[z-y_{0}\right]}{\sigma_{x}^{2}+\sigma_{y}^{2}}$. Formulas for supremum arguments are symmetrical. This follows from commutative property of adding. The reducing of elemental operations number is observed: instead of $\mathrm{O}\left(n^{2}\right)$ amount of adding's and multiplication's operations, where $n$ is the quantity of points in MF's discretization for operands $x$ and $y$, we have got $\mathrm{O}(n)$ operations of both types. It is always appropriate to use preliminary constants calculating: 
$\frac{\sigma_{y}^{2} \cdot x_{0}-\sigma_{x}^{2} \cdot y_{0}}{\sigma_{x}^{2}+\sigma_{y}^{2}}$ and $\frac{\sigma_{x}^{2} \cdot y_{0}-\sigma_{y}^{2} \cdot x_{0}}{\sigma_{x}^{2}+\sigma_{y}^{2}}$ should be computed only one time as well as coefficients $\frac{\sigma_{x}^{2}}{\sigma_{x}^{2}+\sigma_{y}^{2}}$ and $\frac{\sigma_{y}^{2}}{\sigma_{x}^{2}+\sigma_{y}^{2}}$, which are situated before set value of $z$.

Discrepancy with (1) can appear, when we estimate $\sigma_{y}^{2}$ and $\sigma_{x}^{2}$. Result, which is different to one got by (1), may appear, for example, when the form of MF lateral sides is deflected from Gaussian after some transform. In the most of situations this discrepancies are not important, we can disregard them because of necessity of final error's value's rounding off. For getting estimation from above it is possible to offer to estimate MF, the form of which is deviated from Gaussian, with Gaussian with corresponding value of parameter.

For the operation of multiplication we perform the maximum's search for the expression $\max _{x \cdot y=z}\left[\mu_{x}(x) \cdot \mu_{y}(y)\right]=\max _{x \cdot y=z}\left[e^{-\frac{\left(x-x_{0}\right)^{2}}{2 \cdot \sigma_{x}^{2}}} \cdot e^{-\frac{\left(y-y_{0}\right)^{2}}{2 \cdot \sigma_{y}^{2}}}\right]$. This problem is reduced to analytic solving of 4-th order algebraic equation, which has real coefficients, in radicals. This by DescartesEuler's and Ferrari's methods requires a large amount of multiplication's, adding's operations and extractions of the root. That's why we offer to use another way. We perform some modification of multiplication of number $x$ and $y$, which are placed inside corresponding them supports $S_{x}$ and $S_{y}$ :

$$
x \cdot y=\left[x_{\text {rand. }}+x_{\text {syst. }}\right] \cdot\left[y_{\text {rand. }}+y_{\text {syst. }}\right]=x_{\text {rand. }} \cdot y_{\text {rand. }}+x_{\text {rand. }} \cdot y_{\text {syst. }}+y_{\text {cryy. }} \cdot x_{\text {syst. }}+x_{\text {syst. }} \cdot y_{\text {syst. }} \text {. }
$$

Fuzzy numbers $x_{\text {rand }}$ and $y_{\text {rand }}$ andisfy the condition $\arg \max _{x \in S_{x_{c ⿻ y y .}}(x)}\left[\mu_{x_{c y y u}}(x)\right]=\arg \max _{x \in S_{y_{c o y y}}(x)}\left[\mu_{y_{c y y .}}(x)\right]=0$.

For the operation $x_{\text {rand. }} \cdot y_{\text {rand }}$. we find the maximum of expression $\max _{x \cdot y=z}\left[\mu_{x_{\text {rand. }}}(x) \cdot \mu_{y_{\text {rand. }}}(y)\right]=\max _{x \cdot y=z}\left[e^{-\frac{x^{2}}{2 \cdot \sigma_{x}^{2}}} \cdot e^{-\frac{y^{2}}{2 \cdot \sigma_{y}^{2}}}\right]$. Required maximum is reached in the point of maximum of function $f(x, y)=-\frac{x^{2}}{2 \cdot \sigma_{x}^{2}}-\frac{y^{2}}{2 \cdot \sigma_{y}^{2}}$. To determine the value $x_{\text {rand.max }}$ we make substitution $y=z / x$. Then

$$
\begin{gathered}
\varphi(x, z)=f(x, z / x)=-\frac{x^{2}}{2 \cdot \sigma_{x}^{2}}-\frac{(z / x)^{2}}{2 \cdot \sigma_{y}^{2}}, d \varphi(x, z) / d x=0 . \\
\sigma_{y}^{2} \cdot x+\sigma_{x}^{2} \cdot \frac{z}{x} \cdot\left(-\frac{z}{x^{2}}\right)=0, \sigma_{y}^{2} \cdot x^{4}=\sigma_{x}^{2} \cdot z^{2}, x_{\text {rand.max }} \neq 0 \\
x_{\text {rand.max }}= \pm \sqrt{\frac{\sigma_{x}}{\sigma_{y}} \cdot|z|} .
\end{gathered}
$$

The maximization of first summand is getting with correlations $x_{\text {rand } \text { max }}= \pm \sqrt{\frac{\sigma_{x}}{\sigma_{y}} \cdot|z|}$, $y_{\text {rand } \max }= \pm \sqrt{\frac{\sigma_{y}}{\sigma_{x}} \cdot|z|}$. Other operations of (4) represent:

- fuzzy number and coefficient multiplication (linear transform of support axis);

- addings of fuzzy numbers;

- removal of result support axis; 
Instead of $\mathrm{O}\left(n^{2}\right)$ elementary operations for fuzzy interval's multiplication we have an optimized quantity, which complexity is $\mathrm{O}(n)$ of addings, multiplications and extractions of the root. Degree of saving of time grows when the value of $n$ is on the increase.

We offer to present the dividing operation $\frac{x}{y}$ in the form $\left(\frac{1}{y}\right) \cdot x$. Dividing of operands, one of which is fuzzy number, but another is not, may be made by ordinary transform of support abscissas, i.e. $S_{y_{f}}\left(y_{i}\right) \Rightarrow \widetilde{S}_{y_{f}}\left(y_{i}\right)=\frac{1}{S_{y_{f}}\left(y_{i}\right)}, \forall i$. This would bring to us better results, than if we use optimization formulas of conditional extremum method, and allow to use expressions, that we mentioned above for fuzzy numbers multiplication.

\section{Applications examples:}

After determining of elementary arithmetic operations for fuzzy numbers at the way, that performs conditions (*), we receive whole system of operations, which allows us to carry out various transformations. Having given program library with operations over the fuzzy variables (operations ('+', '-', ' $\times$ ', '/’)) to the user we transfer him an ability to create the self-verified programs, input arguments of which are characterized by the uncertainty presented in the form of fuzzy variables and have the meaning of expert estimates.

As the example of offered framework's using we present the result of getting a corridor of uncertainty for the specified degree of belief for signal's amplitude-frequency characteristic, each point of which are fuzzy numbers.
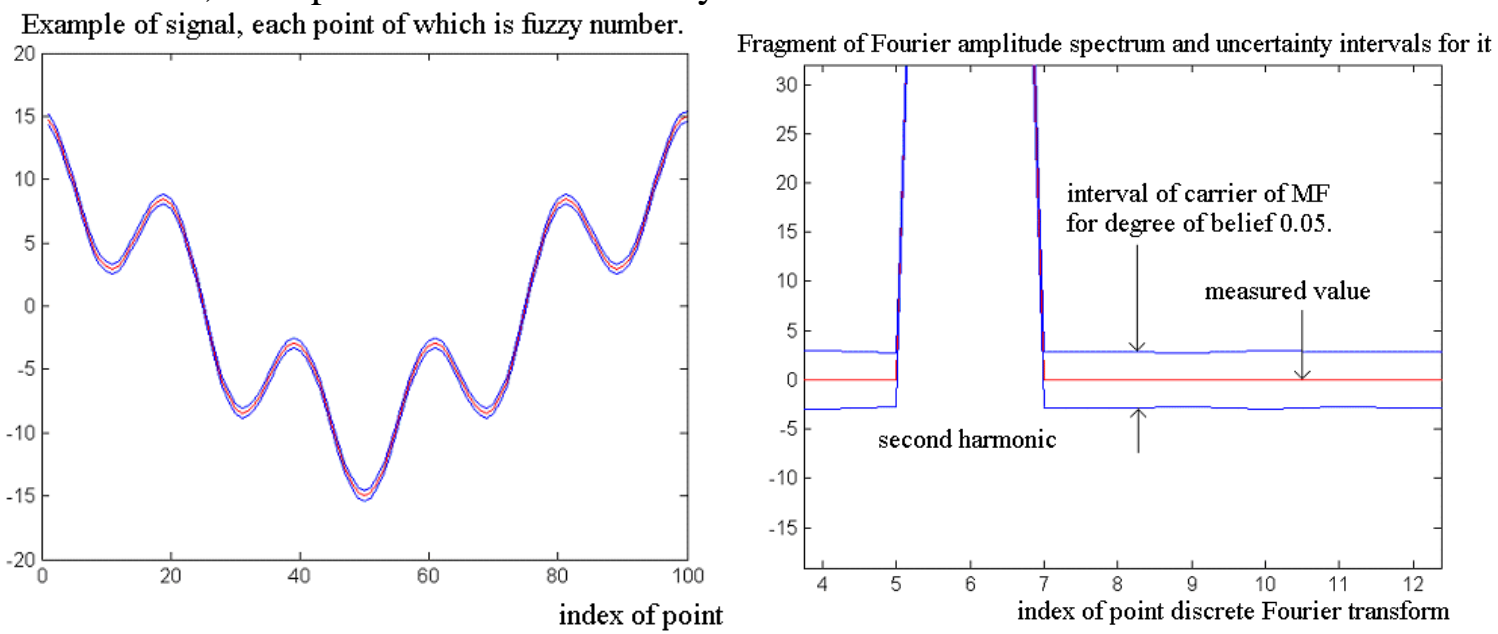

Fig. 3. Example of signal, points of which are fuzzy numbers, and fragment of its Fourier spectrum with uncertainty intervals for degree of belief $\alpha=0.05$.

\section{References}

1. Reznik L. K., Jonson W. C., Solopchenko G. N. Fuzzy interval as a Basis for Measurement Theory // Proc NASA Conf. NAFIPS’94. - San-Antonio, Texas, 1994.

2. Borisov A. N. Uncertain Information Processing in decision making systems // Radio and communications. - Moscow, 1989.

3. Hung T. Nguen, Kreinovich V. Nested intervals and sets: concepts, relations to fuzzy sets, and applications, // Application of Interval Computation / Ed. R. B. Kearfott and V. Kreinovich. - Dordrecht, Boston, London: Kluver Academic Publishers, 1996.

4. Guide to the Expression of Uncertainty in Measurement. 1993. 